\title{
El léxico en las mujeres y los hombres: restricciones inconscientes o culturales
}

\author{
Ginneth Pizarro Chacón \\ Escuela de Literatura y Ciencias del Lenguaje \\ Universidad Nacional, Heredia, Costa Rica
}

\begin{abstract}
Resumen
Definir el léxico de un individuo dependiendo de su género es algo común en la sociedad. Cada hablante ha adquirido un léxico que lo identificará como parte de un género específico y este será utilizado durante toda su vida. Esta forma tan delimitada en el uso de la palabra ha motivado a los estudiosos a investigarlo; por lo tanto, la presente investigación se enfocará en recolectar, analizar y presentar aspectos representativos sobre el uso de la palabra asociada con el género- masculino y femenino- y cómo muchas palabras consideradas tabúes son restringidas a un género específico. Además, se evidenciarán las restricciones psicológicas a que son sometidos los hablantes por parte de la sociedad y cómo muchas de estas normas sociales impuestas están siendo violentadas y cambiadas gradualmente por parte de los hablantes jóvenes. Como parte de esta investigación, se realizó un estudio exhaustivo sobre el tema en cuestión así como un instrumento para la recolección de datos. Este último constó de preguntas cerradas, semiabiertas y abiertas que fueron respondidas por un total de 59 informantes, todos estudiantes de la Universidad Nacional de Costa Rica de entre 20 y 30 años. Entre los resultados más relevantes se enfatiza la visión estereotipada de que los hombres utilizan más palabras vulgares o tabúes que las mujeres. Por lo tanto, las mujeres son asociadas a palabras que enfaticen su feminidad y delicada naturaleza impuestas culturalmente. Otro aspecto relevante es el cambio gradual que ha sufrido la restricción en el uso de ciertas palabras por parte de hombres y mujeres en la sociedad y cómo los hablantes, independientemente de su género, están siendo poco a poco visualizados en igualdad de condiciones lingüísticas. Además, se reafirma que el condicionamiento en el uso de ciertas palabras continúa siendo una imposición cultural inconsciente en el que la familia tiene un papel protagónico. Finalmente, se evidenció que se está dando una revolución léxica gradual entre los hablantes jóvenes debido a un cambio de visión del género.
\end{abstract}

Palabras claves: léxico, géneros masculino y femenino, restricción inconsciente, revolución léxica 


\begin{abstract}
To define an individual's lexicon according to his gender is quite common in our current society. Each speaker has acquired a particular lexicon that will identify him for the rest of his life. This particular view about a speaker's usage of the language has motivated the researchers to investigate about it; consequently, this investigation was focused on collecting, analyzing and presenting representative aspects about the restriction of some taboo words according to the gender. Besides, some psychological restrictions imposed by society as well as gradual changes in youngsters' lexicon are shown as part of the findings. An exhaustive literature review about the subject matter to support the theoretical part was done. To collect the data, a questionnaire was used; it included closed, semi- open and open questions that were asked to 59 students, men and women, between the ages of twenty and thirty from the Universidad Nacional de Costa Rica. As part of the data analysis, it could be mentioned that there is a clear and perpetuated idea that men use more vulgar o taboo words than women. Women are seen as delicate and feminine according to society imposed norms and view. Another relevant aspect is the gradual change in the speakers' linguistic conditions; they are allowed to use the lexicon in a more relaxed and free way. It is remarked that the linguistic conditioning is the result of a cultural imposition that is perpetuated by the family. Finally, a lexical revolution is clearly taking place among young speakers who see a slight change in the way society is visualizing the gender.
\end{abstract}

Palabras claves: lexicon, masculine and feminine gender, unconscious restriction, lexical revolution

\title{
Introducción
}

$\mathrm{P}$ ensar acerca de lo que distingue a un hombre de una mujer es más que visualizarlos dependiendo de su sexo y rol social. Cada grupo en particular tiene características especiales que lo hacen único e irrepetible. Este aspecto incide de manera importante en la forma en que ambos son visualizados por la sociedad. A lo largo de la historia, el género ha jugado un papel fundamental en el comportamiento humano, el desarrollo de sus ideas y el uso de la lengua. Por lo tanto, cada individuo adquiere una serie de normas sociales que le indican lo que se espera o no de él, como individuo activo de la sociedad en que se desarrolla. Esta expectativa moldea su carácter y forma de comportamiento. Es así como el individuo aprende y desarrolla comportamientos y hábitos, tanto culturales como lingüísticos de manera inconsciente. Este estado de aprendizaje inconsciente lo lleva a repetir patrones y a aceptar reglas que le restringen el desarrollo de ciertas actividades así como le definen el uso de la lengua dependiendo de las circunstancias, el entorno social y el género. Este último es uno de los factores más relevantes en este tipo de reglas establecidas e impuestas por la 
sociedad. Por lo tanto, hablantes de ambos sexos -femenino y masculino- son restringidos a utilizar ciertas palabras, como una forma de identificación de género y aceptación social. Esta aceptación conlleva a monitorear su léxico, el lugar donde lo utiliza y a cuidar que éste sea representativo de su género.

\section{El género: aspectos históricos}

A través de los años, muchos estudiosos de la relación mujer-hombre han tratado de explicar la forma en que ambos sexos determinan su rol social y lingüístico, en un mundo cada vez más cambiante y demandante. Sin embargo, proveer una respuesta que satisfaga a ambas partes no ha sido posible. Tanto las feministas como los defensores de los derechos de los hombres han mantenido una lucha ideológica por manifestar su visión de género. Joan W. Scott (1996) en su artículo "El género: una categoría útil para el análisis histórico", manifiesta lo siguiente:

"Género", como sustitución de "mujeres"se emplea también para sugerir que la información sobre las mujeres es necesariamente información sobre los hombres, que un estudio implica al otro. Este uso insiste en que el mundo de las mujeres es parte del mundo de los hombres, creado en él y por él. Este uso rechaza la utilidad interpretativa de la idea de las esferas separadas, manteniendo que el estudio de las mujeres por separado perpetúa la ficción de que una esfera, la experiencia de un sexo, tiene poco o nada que ver con la otra. Además, género se emplea también para designar las relaciones sociales entre sexos. (p. 6)

Esta visión de género no hace referencia a las diferencias existentes entre hombres y mujeres ni a la forma en que su mundo social y lingüístico varía, dependiendo de la cultura y la posición de ambos en la sociedad. Ambos sexos son visualizados como seres que comparten los mismos deseos, visión del mundo y derechos, así como las mismas responsabilidades. Sin embargo, la relación hombre-mujer no está regida por un mismo sistema de derechos y opciones, más allá de que una sociedad aparentemente brinde a todos sus miembros igualdad de oportunidades.

Otra visión es el género sociolingüístico que se define "como un tipo especial de aproximación a la covariación existente entre lengua y género, el cual tiene por objetivo fundamental describir el uso lingüístico, más exactamente los diferentes usos lingüísticos de los hombres y las mujeres como hablantes (Harold Andrés Castañeda Peña y Sandra Teresa Soler Castillo, p.2). Esta última definición permite establecer las diferencias lingüísticas entre el hombre y la mujer; así, se puede evidenciar que las mujeres son las mayores generadoras de cambio en el vocabulario de una sociedad. Esto se debe a que ellas introducen más cantidad de palabras y se arriesgan más al utilizarlas. Por ende, éstas son trasmitidas a sus hijos o grupo cercano. 
Jorge Lemus (2001) señala en su artículo "Sexismo en el lenguaje: mitos y realidades":

es la sociedad la que moldea el uso del idioma, haciendo que éste refleje su cosmovisión. Nótese que no afirmo que la sociedad moldea la estructura del idioma, sino el uso. Con este giro conceptual, se despoja al idioma de toda culpa por sus rasgos sexistas ya que la única responsable de cualquier rasgo sexista en el lenguaje es la sociedad; es decir, nosotros, los usuarios del idioma. (p.12)

Esta aseveración hace pensar que las mujeres y los hombres son quienes establecen e imponen el uso de determinadas palabras o frases, de acuerdo con su género y rol en la sociedad. Por lo tanto, surge una pregunta al respecto:¿Por qué ambos sexos se consideran afectados por el uso de la lengua y la imposición de reglas en su uso si ellos pueden establecer cambios y defender el libre uso de la palabra?

El hablante es responsable del uso de la palabra y debería regir su uso con base en criterios de oportunidad y contexto; sin embargo, la situación lingüística de una sociedad no se rige por la escogencia individual, sino por "la igualdad de la mujer con el hombre (que) nace del control de los medios de subsistencia, a mayor control de la mujer de los recursos estratégicos de la comunidad, mayor igualdad con el hombre, y a menor control de estos recursos mayor subyugación de la mujer" (Lemus, 2001, p.13). Por lo tanto, el control lingüístico de los hablantes está establecido por el poder y la representación de éste en la sociedad. En el momento en que este poder es amenazado por un cambio en la cuota de poder de las mujeres y éstas “cambia(n) su estatus social y tiene(n) acceso al poder" (p.13), el uso de la lengua cambia en una forma asombrosa. Este cambio en el poder y el uso del poder mismo, da como resultado que los hablantes sean capaces ya sea de realizar cambios o de perpetuar patrones lingüísticos que restringen el libre uso del léxico en las conversaciones diarias. Al inicio, los estudios de género

partieron de la premisa de que como hombre y mujer no hablan de la misma manera (hombres y mujeres hacen usos particulares de la lengua), la mujer no se ajustaba al buen hablar y poseía comportamientos lingüísticos distintos de los del hombre; por tanto, eran estos comportamientos los que debían explicarse. (Harold Andrés Castañeda Peña y Sandra Teresa Soler Castillo, p.3)

Por lo tanto, el uso de la lengua no era visualizado como una imposición cultural, sino que era determinado por los comportamientos lingüísticos del hablante según su género.

Cabe mencionar que esta visión segregada del género es también manifestada en los diccionarios, fuentes de consulta para aclarar dudas sobre la palabra y su uso por parte del hablante. En este, "el papel ético del lexicógrafo, quien puede trasladar a su creación mensajes subliminales y enunciados manipuladores, de 
una u otra tendencia o postura ideológica" (Aurora M. Camacho Barreiro, 2004, p.32), no deben ser visualizados con ligereza. Estos pueden trasmitir mensajes mediante "definiciones sesgadas o ideológicamente condicionadas en material de política, de género, de raza, entre otras parcelas de la vida de una sociedad" (p.32). Por lo tanto, una palabra o frase puede ser definida de acuerdo con la visión de mundo y del rol que la mujer o el hombre tiene en la sociedad. Estos aspectos pueden incidir positiva o negativamente en el derecho a la palabra, el condicionamiento de su uso en contexto o su prohibición según el género.

\section{Diferencias léxicas: adquisición inconsciente}

$\mathrm{Al}$ inicio del proceso de aprendizaje, todos los hablantes comparten los mismos patrones sintácticos, fonológicos y semánticos; sin embargo, estos hablantes son guiados a utilizar un léxico restringido de acuerdo con su género y contexto. Este proceso de aprendizaje es realizado en una forma inconsciente desde la infancia. El deseo de los niños de imitar el vocabulario de sus padres o madres nace de su visualización como modelos que determinan su rol lingüístico y lo que deben o no deben decir. De esta forma, los patrones sociales y lingüísticos son asignados desde el inicio del proceso de adquisición de la lengua materna; además, el repertorio verbal será determinado no por aspectos tangibles sino mentales.

La falta de acceso de la mujer a la educación y su confinamiento al ámbito hogareño influyen directamente en su hablar, no solamente en lo que respecta a un léxico limitado, sino también al tipo de temas de conversación. Esto nos indica que para que un cambio en los patrones lingüísticos de las mujeres ocurra, ellas deben tener acceso a la educación en los mismos términos que los hombres. Probablemente sea en el hogar donde se requiere cambiar los paradigmas que han mantenido a la mujer fuera de la sociedad productiva. Los padres de familia se encargan de perpetuar los roles del hombre y la mujer en la sociedad, a través de la educación de sus hijos: tareas en el hogar, juguetes, castigos, ejemplos, libertades y otras actividades son diferenciadas por el sexo de los hijos (Aurora M. Camacho Barreiro, 2004, p.15). Además, las mujeres continúan teniendo menos oportunidades laborales y son confinadas a un entorno hogareño que limita su desarrollo en los patrones lingüísticos y en el uso de la lengua.

\section{La utilización apropiada de las palabras: una adquisición cultural}

Cuando el individuo está en su proceso de adquisición de la lengua, ésta es trasmitida de acuerdo con las normas gramaticales y reglas establecidas en el sistema de cada una. Por lo tanto, los hablantes aprenden los mismos patrones gramaticales y uso de las palabras en el contexto apropiado. Sin embargo, estos patrones no limitan al hablante a utilizar una determinada palabra o frase; este es el resultado de una adquisición cultural que determina lo que se puede o debe utilizar por parte del hablante según su género o posición en la sociedad. Desde 
el inicio de su proceso de adquisición de la lengua, los niños son enseñados a diferenciar el léxico utilizado en la escuela, la casa, los lugares públicos o con sus amigos. Si alguno de estos hablantes quebranta las reglas impuestas, será considerado como perteneciente al género opuesto y será rechazado moralmente por su familia, amigos o desconocidos.

Un ente que cumple un papel protagónico en la apropiada utilización de las palabras y promueve esta adquisición cultural es la escuela. Esta "tiene un cierto margen de maniobra: puede contribuir a reforzar la desigualdad, a consolidar las estructuras jerárquicas de la sociedad" (Amparo Tusón Valls); por lo tanto, el individuo es enseñado desde su infancia a no cuestionar las normas impuestas, sino a acatarlas sin ningún tipo de cuestionamiento. Así, la división imaginaria entre el léxico que deben utilizar los hombres y las mujeres se perpetúa de una generación a otra.

\section{Hombres y mujeres: formas distintas de hablar}

Cuando se analiza el discurso de un hombre y el de una mujer, este está determinado por varios aspectos: el tema discutido, el género de los participantes, el contexto donde se realiza la conversación y el estilo en el habla. Durante una conversación, el tema por discutir representa un factor relevante ya que determina el número de intervenciones de los participantes en la conversación. Así, la escogencia de un tema que se refiera a aspectos femeninos o masculinos será relevante para que tanto el hombre como la mujer se sientan cómodos para expresar sus ideas libremente. Lemus (2001) indica que

El hombre, por ejemplo, es directo en su hablar, trata de dirigir la conversación y los temas a discutir, decide cuándo terminar una conversación o cuándo cambiar de tema. Además, por estar en contacto con otras personas para negociar sus productos, su léxico aumenta y su hablar se ve influenciado por el medio. La mujer, por su lado, se queda en casa y la extensión de su vocabulario se limita a los asuntos del hogar, por lo que en las conversaciones intersexuales tienen un rol más pasivo. (p.15)

Esto ciertamente reduce el número de temas que las mujeres y los hombres discuten cuando están con otros o comparten entre ellos.

El género de los participantes es otro factor determinante en el uso de la lengua. En una conversación entre hablantes de diferente género en que la mayoría son hombres, las mujeres tienen poca participación o ninguna. El sexo masculino será el que determine no solamente el tema, sino el número de intervenciones en la conversación. Otro aspecto relevante es el contexto donde se realiza la conversación; un entorno donde tanto el hombre como la mujer se sienten cómodos para expresar sus ideas permitirá que ambos utilicen la lengua con una mayor libertad. Además, Trudgill (2000) enfatiza que "muchas sociedades esperan que las mujeres demuestren un mayor nivel de adhesión a las normas sociales- un 
mejor comportamiento- que los hombres" (p.73). Finalmente, el estilo utilizado, formal o informal, limita o permite el uso de un léxico más restringido y apegado a lo establecido por la sociedad, o por el contrario, brinda la libre escogencia de este y su uso sin restricciones de género.

\section{Justificación del estudio}

A través de la historia, la sociedad ha establecido una serie de normas y restricciones como una forma de mantener el orden establecido y lograr la convivencia. Este orden ha estado regido por obstáculos geográficos, físicos, ideológicos y lingüísticos. Este último será el tema relevante en esta investigación por ser un aspecto intrínseco al ser humano, así como una herramienta capaz de cambiar mentes y trasmitir ideas. La lengua es visualizada como un sistema compuesto por aspectos fonológicos, morfológicos, sintácticos, semánticos y léxicos. Este último muestra una gran variabilidad dependiendo de factores tales como zona geográfica, nivel educacional, posición económica, grado académico y género. No obstante, el género es el aspecto desde el que se enfocará este estudio, ya que está ligado al léxico utilizado por hombres y mujeres; este léxico ha sido preestablecido e impuesto a los hablantes como norma por parte de la sociedad. Por lo tanto, el género del hablante restringirá el uso de determinadas palabras en público, en la intimidad de su casa o en las relaciones con sus amigos o conocidos.

\section{Propósito del estudio y preguntas de la investigación}

El propósito primordial de esta investigación es analizar y presentar aspectos relacionados con el tipo de palabras asociadas con el género- femenino y masculino- los cuales son prohibidos o tabúes en ciertas sociedades, así como la discriminación por parte de otros si el hablante viola lo establecido por la norma social. Otro aspecto relevante es evidenciar las restricciones psicológicas a las que son sometidos los hablantes por parte de la sociedad como forma de ser aceptados en un mundo cada vez más influenciado por las apariencias y formas de comportamiento. Finalmente, se pretende explorar la forma en que los jóvenes y adultos jóvenes violan las normas sociales en el uso del léxico como una forma de libertad de expresión. Siendo la restricción en el uso de la palabra uno de los aspectos más evidentes para el hablante, surgen algunas preguntas:

1. ¿Determina el género el léxico de un individuo?

2. ¿Tiene la palabra el poder de agrupar individuos de acuerdo con su género?

3. ¿Son las restricciones e imposiciones en el uso de la palabra una forma de controlar a los hablantes por parte de la sociedad?

4. ¿Se adaptan el hombre y la mujer a las normas en el uso de la palabra en una forma consciente o inconsciente? 
5. ¿Cuáles son las consecuencias negativas de violar dichas normas?

6. ¿Están las nuevas generaciones produciendo un cambio en su léxico como forma de manifestación de su libertad de expresión y liberación?

\section{Metodología: participantes e instrumento para recolectar la información}

El presente estudio se enfocará en el léxico utilizado por los hombres y las mujeres y cómo este está influenciado/mediado por restricciones psicológicas y sociales; la investigación estará dividida en dos partes.

La primera abarca una investigación exhaustiva sobre el uso de la palabra de acuerdo con el género, las normas que lo rigen y las restricciones léxicas impuestas por la sociedad, con el objetivo de evidenciar estudios que se hayan escrito al respecto sobre el tema. Además, se pretende corroborar si el tema propuesto podrá aportar nueva información sobre los cambios en el habla de la población actual.

La segunda etapa presenta un instrumento con preguntas sobre el tema, el cual abarca aspectos tales como rasgos culturales, léxicos e implicaciones negativas en el uso de palabras restringidas según el género del hablante. El uso de este cuestionario permitirá obtener información confiable para corroborar la aseveración sobre las restricciones en el uso de la palabra y cómo éste es determinado por el género del hablante. Este instrumento será aplicado a estudiantes de entre 20 a 30 años con el objetivo de comprobar si las normas sociales determinan el uso de la palabra, si estas restricciones son aceptadas en forma consciente y si el cambio en el uso del léxico está determinado por el grupo generacional.

\section{Resultados}

La obtención de resultados veraces sobre aspectos relacionados con las palabras o expresiones que distinguen a un hombre de una mujer no es una tarea fácil. En la mayoría de los casos la forma como se plantea la pregunta o el criterio de quien la contesta determinará los datos obtenidos y cómo estos serán interpretados por el investigador. En este estudio se utilizó un cuestionario con preguntas cerradas, semicerradas y abiertas, el cual permitió que los informantes pudieran expresar sus criterios acerca de un aspecto en particular en una forma guiada en parte del cuestionario y en una forma abierta de acuerdo con lo que creían en una segunda parte de este. Por lo tanto, se utilizó un cuestionario que estaba dividido en dos partes: rasgos culturales y léxico. Este fue completado por 59 informantes con edades de entre 20 y 30 años, estudiantes de la Universidad Nacional de Costa Rica, sede de Heredia. A continuación se procederá a brindar los resultados de dicho trabajo de campo y como este reafirma la línea imaginaria impuesta inconscientemente por la sociedad en el uso del léxico por parte de las mujeres y los hombres.

En relación con los rasgos culturales, 56\% de los informantes mencionaron que el principal responsable económico de la familia es el hombre, lo cual perpetúa 
el estereotipo cultural del hombre como proveedor del hogar. Un 29\% indicó que tanto el hombre como la mujer son responsables del soporte económico del hogar y un $15 \%$ visualiza a la mujer como la responsable económica del hogar. Además, un $46 \%$ mencionó que tanto el hombre como la mujer toman las decisiones importantes en la familia, mientras que un $39 \%$ señala a la mujer como pieza fundamental en esa toma de decisiones. Solo un $15 \%$ mencionó al hombre como el tomador de decisiones importantes.

Otro aspecto importante es que el $71 \%$ de los informantes indica que la mujer es la principal responsable del cuido de los hijos, de asistir a las reuniones escolares, así como de la crianza en general. Solamente un $29 \%$ señala a ambos géneros como responsables de dichas actividades y ninguno mencionó al género masculino como principal responsable. Al preguntárseles a quién consideraban el jefe de la familia, $34 \%$ indicaron que el hombre sigue siendo visualizado como el centro de la familia. Sin embargo, otro $34 \%$ también visualiza tanto al hombre como a la mujer como cabezas de familia y un $32 \%$ considera a la mujer como el género a cargo del hogar. Esta visión de que ambos géneros pueden representar el rol de jefes de familia proyecta un cambio significativo en la sociedad costarricense. La imagen tradicional de hombre como cabeza de la familia ha cambiado y gradualmente ambos asumen un protagonismo en este aspecto.

En cuanto a quién se asocia socialmente con un ser emocionalmente débil, sensible y dócil, el 88\% de los informantes señala a la mujer como epítome de la fragilidad en nuestra sociedad. Por su parte, el $73 \%$ señala al hombre como un ser asociado al rol de individuo fuerte y racional.

En relación con las palabras que distinguen a una mujer de un hombre y viceversa, los informantes indicaron que los hombres utilizan más palabras vulgares y coloquiales tales como mae, tuanis, pura vida, güevón, cabra, maldita, perra, calidad, puta, hijueputa, playo, hijo de puta, bonito, interesante, rata, malparido, cara de picha, guineo, zorra, idiota, compa, va a hartar, pichudo, pepino (golazo) y compa. Mientras que las mujeres usan palabras como: diminutivos (bonito, cosito), lindo, linda, hermoso, sensible, tierno, romántico, gracias, chicas, chicos, amor, dulzura, ternura, cariño, precioso, delicioso, cosi, bello, corazón, bella, dulce, adorable, amor, mi amor, cosita, hermoso, muchacho va a comer, divino, corronga, amiga, amoroso, mae y te amo.

En toda sociedad existen palabras que han sido restringidas a un grupo en particular de acuerdo con su género. En relación con esto, al preguntárseles a los informantes acerca de las palabras que son prohibidas a las hablantes femeninas, mencionaron las siguientes: mae, pichudo, hijo de puta, maldito (a), mierda, puta, malparido, picha, playo, marica, güevón, idiota, vara, estupidez, cagar, idiota, mitata, mimama, tuanis, verga, despichar, harina (plata), perra, rata, chiva, chuzo, tuanis, zorra, mongolo y carepicha.

Asimismo, ellos indicaron palabras o frases que son prohibidas a los hablantes masculinos. Se mencionaron: precioso, hermoso, lindo, bonito(a), tierno, belleza, ¡Qué bonito!, ¡Qué tierno!, dulce, romántico, sensible, querido(a), adorable, "cute", cariño, cielo, divino, tesoro, agitado, estúpido, desgraciado, concho (rudo), bella, "sweetie", amor, corrongo, guapo, cosi, cariño, corazón, "se ve muy bien 
mae”, "Qué lindo”, ¿Estoy gordo?, ¡Ay!, todo lindo, cosita, guapo, príncipe, guapo (apuesto), triste y ¡Muy lindo!.

Otro aspecto importante que se evaluó fue identificar las razones por las cuales la restricción en el uso de ciertas palabras por parte de hombres y mujeres en la sociedad ha cambiado paulatinamente. Entre las posibles razones, un 54\% mencionó que la igualdad de género ante la sociedad ha contribuido a visualizar a los hablantes como seres iguales sin importar su género. Un 53\% visualiza también el acceso de la mujer a la educación y el trabajo fuera del hogar como elementos que han eliminado las barreras para utilizar el léxico libremente. Un $47 \%$ señala que el cambio se debe al uso de la palabra como un indicador de la libertad de expresión del individuo. Finalmente, un $22 \%$ cree que la visión del hombre como un ser sensible y amoroso ha contribuido a esta apertura en el uso de la palabra.

En cuanto a si la restricción en el uso de ciertas palabras según el género es una imposición cultural al hablante, los informantes manifestaron que la cultura define a sus individuos dependiendo de su zona geográfica y raíces culturales. Además, se menciona que la visión de las mujeres está relacionada con el hecho de ser tiernas, dulces, amables y que deberían permanecer en el hogar; el hombre, por el contrario, debe ser tosco y fuerte en su habla. Esta visión masculina del uso de la palabra por parte del hombre es impuesta por el género masculino principalmente, lo cual evidencia que se trata de una sociedad machista y patriarcal; esto a su vez es transmitido a los hijos varones. Por lo tanto, se reafirma que este condicionamiento en el léxico de ambos géneros es aprendido desde la infancia, principalmente en el hogar. Cabe mencionar que se indica que, en algunas ocasiones, el género femenino se restringe en el uso del léxico por seguir un condicionamiento social. Además, se señala que existe una gran cantidad de estereotipos que encasillan a ambos géneros en un uso particular de la unidad léxica.

Otro aspecto mencionado es la discriminación por parte de la sociedad cuando un hablante utiliza la palabra que le es prohibida. También llama la atención que se menciona la religión como un ente controlador, que define en forma indirecta lo que se debe utilizar y quién lo utiliza. Se menciona la no aceptación por parte de la sociedad de que la mujer y el hombre tengan roles similares y se desarrollen lingüísticamente en forma igualitaria. Algunos informantes consideran que en muchos casos los hablantes utilizan la palabra como quieren y cuando quieren, y así pueden seguir sin restricciones sociales o culturales. Sin embargo, los hablantes que violen las restricciones impuestas son considerados incultos, maleducados o de inclinación sexual dudosa. Además, se manifiesta que esta restricción en el uso de la palabra restringe la libertad de expresión sin importar el género. Se menciona que los "tabúes léxicos" son mecanismos que la sociedad utiliza para imponer roles y perpetuarlos.

Otro aspecto importante sobre el que se les preguntó fue el relacionado con las razones que se dan para que la restricción en el uso de la palabra sea un fenómeno inconsciente. Un 68\% manifestó que es debido a un proceso de adquisición de normas sociales y lingüísticas adoptadas como propias; por lo tanto, el individuo asume que esta es la única forma de expresarse y no asume un cambio 
en el uso de la palabra. Un 49\% considera que un aprendizaje progresivo desde la infancia ha influido en asumir como propias restricciones impuestas por los padres, educadores o adultos mayores con quienes se relacionan. Un 37\% indica que una forma de imitación del lenguaje utilizado por los padres y madres es primordial en perpetuar las diferencias léxicas dependiendo del género. Los hablantes jóvenes, niños y preadolescentes tienden a imitar la forma de comportamiento y uso léxico de sus padres como modelo único.

Finalmente, al preguntárseles si las nuevas generaciones están cambiando esta norma e iniciando una revolución léxica, recalcaron que ahora se deja al niño ser más sensible y a la mujer ser más fuerte. Además, al existir más variabilidad léxica, los hombres y las mujeres pueden usar las mismas palabras sin afectar su imagen o condición social. Debido a las ideas de igualdad de género, tanto la mujer como el hombre utilizan el léxico deseado o escogido en sus lugares de estudio y hogares. Es relevante recalcar que los jóvenes son los hablantes que utilizan el léxico en igualdad de condiciones de acuerdo con el contexto social. Otro aspecto importante es que las personas quieren igualdad en todo y expresarse libremente. Esto último es especialmente influenciado por la tecnología y las redes sociales las cuales facilitan que los hablantes se relacionen entre sí. Cabe recalcar que se indica que la sociedad está cambiando paulatinamente debido al proceso de globalización como una forma de reivindicar a ambos géneros y sus roles en la sociedad. La equidad de género es también reflejada en el libre uso del léxico. Se manifiesta que las nuevas generaciones piensan diferente y no siguen las mismas creencias que sus padres o hermanos. Se hace referencia a que el ambiente, tanto por influencias internas como factores externos (otras culturas) que nos rodean y la inclusión de nuevas palabras a nuestro vocabulario, han cambiado las normas en relación con este aspecto. Además, se indica que no es solamente una revolución léxica, sino también una evolución. Las lenguas están en constante cambio, pero esos cambios no son necesariamente premeditados; son parte de la naturaleza del lenguaje. La gran influencia de los medios de comunicación dicta en gran medida cambios en el léxico. Sin embargo, algunos entrevistados indican que una gran parte de la población todavía está muy arraigada a las normas culturales y lingüísticas. Se menciona que muchas palabras pueden cambiar, pero siempre habrá términos prohibidos para uno u otro género.

\section{Discusión}

$\mathrm{Al}$ analizar detenidamente los resultados de la encuesta realizada, esta investigadora reafirma algunas de las aseveraciones que se han hecho en otros estudios sobre la restricción en el uso del léxico dependiendo del género del hablante. Por lo tanto, se procederá a mencionar algunos aspectos que confirman tal aseveración.

Primero, un número significativo de informantes indica que tanto hombres como mujeres son responsables del soporte económico de la familia. Esto indica que en la actualidad, la visión del hombre como único proveedor económico ha 
ido cambiando. La mujer es vista como un ser posicionado en el mercado laboral y un gran apoyo económico para la familia. No obstante, veremos que esta situación no se ve reflejada en su libertad de uso de la palabra.

Segundo, la mujer sigue siendo la persona responsable del cuidado de los niños, lo que lleva a pensar que no obstante su aporte económico, ella sigue teniendo una gran duplicidad de funciones en el hogar. Tercero, un grupo importante de informantes considera a ambos géneros como jefes de familia que deben asumir responsabilidades en igualdad de condiciones. En algunas sociedades las mujeres son cabeza del hogar, por lo tanto, dichas sociedades no imponen restricciones en el uso de la palabra. Cuarto, a pesar de que la mujer tiene un rol importante como proveedora económica del hogar y responsable de la crianza y cuidado de los hijos, continúa siendo visualizada como un ser emocionalmente débil y vulnerable. Por otro lado, el hombre no pierde su rol de ser dominante y pensante. Esto ciertamente es injusto para ambos géneros ya que tanto el hombre como la mujer poseen características que muestran sus áreas sensibles y humanas y sus áreas fuertes y dominantes. Se brinda una perspectiva errónea de ambos sexos, lo cual no permite que puedan desarrollar y demostrar esas áreas en su léxico.

Quinto, las palabras que distinguen a una mujer de un hombre evidencian esa perpetuación machista por parte de ambos sexos. El hombre solamente es identificado como usuario de palabras vulgares y toscas; no se le permite utilizar palabras dulces y suaves que expresen sus sentimientos. Si las utiliza, es catalogado de homosexual o débil, característica rechazada por la sociedad. Definitivamente, muchas palabras pueden cambiar, pero siempre habrá términos prohibidos para uno u otro género. Sexto, las palabras tabúes son parte de nuestra cultura centroamericana, la cual dicta los roles de la mujer y el hombre que influyen en su léxico. Así, un hombre que no use palabras vulgares relacionadas con el sexo o partes del cuerpo no es considerado macho y representante de su género. Así mismo, una mujer que no se manifieste dulce, maternal y cuidadora es rechazada o criticada por otros. Ambos géneros son presionados a representar un rol léxico establecido por las normas sociales sin importar su libertad de expresión o ideas.

Sétimo, ciertamente la restricción en el uso de ciertas palabras por parte de hombres y mujeres en la sociedad ha cambiado un poco. La visión actual sobre la igualdad de género ha propiciado una apertura lenta hacia el cambio. La mujer se ha posicionado en un rol más allá de ser madre, esposa e hija. Este empoderamiento de la mujer ha permitido que exija una mayor equidad en su forma de expresar ideas, el uso de la lengua y el respeto a la diversidad léxica sin importar el género del hablante. Además, el hombre lentamente asume roles protagónicos en el hogar y expresa sus debilidades en una forma más abierta. Esto conlleva un uso de palabras menos ligadas a su género, pero más cargadas de emotividad y sentimientos.

Finalmente, se está dando una revolución léxica por parte de las nuevas generaciones, como lo evidencian los informantes, quienes exponen su capacidad de utilizar muchas de las palabras hasta hace poco prohibidas para su género como parte de su léxico cotidiano. Esto ha ayudado a abrir puertas a la libre y productiva utilización de la palabra por parte de cualquier hablante sin importar su género. 


\section{Conclusiones}

Definitivamente el léxico en las mujeres y los hombres es un tema que merece ser discutido. El léxico de un individuo no debe ser un factor que identifique, estereotipe o discrimine a ningún hablante y mucho menos el género debe tener un rol predominante en este uso. Sin embargo, lamentablemente el género ha sido un factor determinante en el uso de la palabra. Hablantes de ambos sexos han sido sometidos a restricciones tanto inconscientes como culturales que determinan su léxico tanto en el ámbito público como privado. Inconscientemente, se nos ha enseñado a utilizar ciertas palabras o a evadir aquellas consideradas tabú. Los "tabúes léxicos" son mecanismos que la sociedad utiliza para imponer roles y perpetuarlos. Si alguna vez osamos violentar las restricciones impuestas por las normas sociales, seremos considerados incultos, maleducados o de inclinación sexual dudosa.

Otro aspecto importante es el hecho de que el hablante adquiere estas restricciones en el habla por medio de la imitación del lenguaje utilizado por los padres y las madres, lo cual es primordial en perpetuar las diferencias léxicas dependiendo del género. Los hablantes jóvenes, niños y preadolescentes tienden a imitar la forma de comportamiento y uso léxico de sus padres como modelo único. La discusión anterior nos lleva a asegurar que el problema del sexismo en el lenguaje no es lingüístico sino sociocultural. Todos tenemos derecho a expresarnos de la manera que queramos de acuerdo con nuestra educación y estilo de vida. Por lo tanto, la igualdad en el uso de la palabra no debe atentar contra la dignidad de los demás.

La escogencia de la palabra está determinada por la aceptación del oyente. Esta aseveración está apoyada por el hecho de que muchas veces para escoger nuestras palabras nos basamos en el qué dirán o qué imagen o visión de mí como hablante tienen los demás. Sin embargo, deberíamos preocuparnos más por cómo expresamos nuestras ideas y si los oyentes comprenden nuestro mensaje. Amparo Tusón Valls (1996) indica que

en lo que atañe a los individuos, el repertorio verbal se refiere al conjunto de recursos lingüísticos que los hablantes tienen a su alcance y usan para llevar a cabo sus actividades cotidianas como miembros de su grupo. Como los recursos son variados, se entiende que el individuo selecciona, elige, a la hora de comunicarse, y esa elección se considera significativa en sí misma porque desvela normas de uso colectivas (o transgresiones de las normas de uso). (p.1)

Así, la selección de tal o cual palabra es solamente el medio para comunicarnos, nunca este medio debe determinar mi posición social o aceptación por parte de la sociedad.

Esta visión de comunidad lingüística más abierta al cambio evidencia que debido a las ideas de igualdad de género, tanto la mujer como el hombre están utilizando una diversidad léxica en sus lugares de estudio y hogares. Por 
lo tanto, el lenguaje inclusivo empieza a cobrar relevancia en nuestro mundo lingüístico actual. La apertura de nuevas palabras nos lleva a una forma más integral de comunicarnos y balancear el papel del hombre y la mujer en la sociedad. Tanto mujeres como hombres usan palabras tabúes o palabras dulces; este es un cambio en el que los jóvenes hablantes tienen un rol protagónico. Ellos rechazan la discriminación debido a que están rodeados por una sociedad más diversa y multicultural.

Definitivamente, es necesario educar a los jóvenes sobre la igualdad en el uso del léxico. Por medio de la educación, los hablantes están cambiando los paradigmas. El hablante joven es más liberal y pone como prioridad sus pensamientos y no los que la sociedad le impone. Sin embargo, los hablantes que no tienen acceso a la educación continúan repitiendo patrones en la restricción en el uso del léxico. Nuevamente, Amparo Tusón Valls (1996) expone que

frente al concepto de 'comunidad lingüística' entendida como un todo homogéneo por estructuralistas y generativistas, aparece el concepto de comunidad de habla, que pone el acento en la organización que se establece entre los miembros de un grupo a través de las redes de comunicación social e interindividual. Una de las características de la comunidad de habla será, también, la diversidad, la heterogeneidad, tanto en el plano sociocultural o económico como en el plano lingüístico. (p.1)

La diversidad brinda apertura a nuevas ideas y cambios en el léxico; así, a mayor participación de la mujer en la sociedad en términos igualitarios con el hombre, menores rasgos sexistas antropocéntricos en el uso de la lengua (Lemus, 2001, p. 12). Finalmente, mientras esta relación de desigualdad entre el hombre y la mujer exista, seguirán existiendo diferencias en el habla y el uso del lenguaje seguirá siendo antropocéntrico (p.15).

\section{Bibliografía}

Camacho Barreiro, Aurora M. (2004). De cómo los diccionarios reflejan la sexualidad y otros conceptos afines. IL Anuario, 35.

Castañeda Peña, Harold Andrés y Sandra Teresa Soler Castillo. (s.f.) Universidad Pedagógica Nacional. Recuperado de http://www.pedagogica. edu.co/ storage/ folios/ articulos/folios09_05arti.pdf

Lemus, Jorge E. (2001). Sexismo en el lenguaje: mitos y realidades. Publicado en Memorias del Encuentro de la Red Centroamericana de Antropología. Asociación Salvadoreña de Antropología, San Salvador. Recuperado de http://hispanismo.cervantes.es/documentos/11773-Sexismo\%20en\%20el\%20 lenguaje-1.pdf

Scott, Joan W. (1996). El género: una categoría útil para el análisis histórico. Recuperado de http://www.cholonautas.edu.pe/modulo/upload/scott.pdf 
Trudgill, Peter (2000). Sociolinguistics: An introduction to language and society. $4^{\mathrm{a}}$ ed. Londres: Penguin Books.

Tusón Valls, Amparo (1996). Iguales ante la lengua, desiguales en el uso. Bases sociolingüisticas para el desarrollo discursivo. Recuperado de https:// campus virtual.univalle.edu.co/moodle/pluginfile.php /207240/mod_resource/content/ 0/Iguales_ante_la_lengua_desiguales_en_el_uso_Tuson.pdf

\section{Anexo 1}

\section{Cuestionario (Informantes)}

Género:

Edad:

Zona

geográfica:

El siguiente instrumento será utilizado para recolectar información sobre el uso de ciertas palabras en el habla de hombres y mujeres. La información recolectada proporcionará suficiente evidencia para reafirmar el hecho de que el uso y la escogencia de la palabra están determinados por el sexo del hablante.

\section{Rasgos culturales}

¿Quién es el/la principal responsable económico(a) de la familia?
a) hombre
b) mujer

¿Quién toma las decisiones importantes en la familia?
a) hombre
b) mujer

¿Quién es el principal responsable del cuido de los hijos, asistir a las reuniones escolares, la crianza en general?
a) hombre
b) mujer

¿Quién es el jefe de la familia?
a) hombre
b) mujer

¿Quién se asocia socialmente con un ser emocionalmente débil, sensible, dócil?
a) hombre
b) mujer

Según su criterio, ¿a quién es asociado el rol de individuo fuerte, racional?
a) hombre
b) mujer 
II. El léxico

Mencione las palabras que distinguen a una mujer de un hombre y viceversa.

Mencione cuatro palabras que son prohibidas a:

las hablantes femeninas

los hablantes masculinos

La restricción en el uso de ciertas palabras por parte de hombres y mujeres en la sociedad ha cambiado debido a:

- El acceso de la mujer a la educación y el trabajo fuera del hogar.

- La igualdad de género ante la sociedad.

- La visión del hombre como un ser sensible y amoroso.

- El uso de la palabra como un indicador de la libertad de expresión del individuo.

Implicaciones negativas

¿Es la restricción en el uso de ciertas palabras dependiendo del género una imposición cultural al hablante? Explique.

La restricción en el uso de la palabra es un fenómeno inconsciente debido a:

- Un aprendizaje progresivo desde la infancia.

- Una forma de imitación del lenguaje utilizado por los padres y madres.

- Un proceso de adquisición de normas sociales y lingüísticas adoptadas como propias.

¿Están las nuevas generaciones cambiando esta norma e iniciando una revolución léxica? Indique por qué

¡Gracias por su ayuda en esta investigación! 